\title{
MHD numerical simulations of Perseus A: formation of filaments and magnetic loops
}

\author{
Diego Falceta-Gonçalves ${ }^{1}$, E. M. de Gouveia dal Pino ${ }^{2}$, \\ J. Gallagher III $^{3}$ and A. Lazarian ${ }^{3}$ \\ ${ }^{1}$ Núcleo de Astrofísica Teórica, Universidade Cruzeiro do Sul, Brazil \\ email: diego.goncalves@cruzeirodosul.edu.br \\ ${ }^{2}$ Instituto de Astronomia, Geofísica e Ciências Atmosféricas, Universidade de São Paulo, Brazil \\ ${ }^{3}$ Astronomy Department, University of Wisconsin, USA
}

\begin{abstract}
The Perseus Cluster (A426) is the brightest cluster of galaxies observed in X-rays in the sky and its giant central galaxy (NGC1275) hosts the extended double radio source $3 \mathrm{C} 84$. There is a spectacular H-alpha nebulosity surrounding NGC1275 with loops and filaments that are probably magnetized and extend over $100 \mathrm{kpc}$. The continuous blowing of bubbles leading to the propagation of shock fronts is also evident and more recently, outflow and infall velocities of several $1000 \mathrm{~km} / \mathrm{s}$ have been detected associated to the surrounding filaments. We here present preliminary results of 2.5D MHD simulations of the Perseus cluster central region assuming that the production of the outflow structures and loops that arise from the surface of NGC1275 are due to turbulent injection triggered by recent star formation and SNe activity in the galaxy. This is in turn, probably induced by a continuous gas infall from the satellite galaxies around NGC1275. Our simulations which include both, the turbulent gas outflow and gas infall from the surroundings, have revealed a continuous formation of the observed features, like the filaments, the gigantic magnetic loops and weak shock fronts that propagate into the ICM medium with the observed velocities of $1000-5000 \mathrm{~km} / \mathrm{s}$. After $10 \mathrm{Myr}$, a nearly steady state is established between the outflow material emerging from the central galaxy and the inflow gas from the surrounds. The outflow activity seems to retard the cannibalism action of the central galaxy over the surrounding galaxies. This result may have important implications over the evolution of the whole cluster as it seems to indicate that the SF and SNe production that are induced by the cannibalism may help to decelerate the later due to turbulence and outflow production. These results also offer important clues to the hot halo formation in the center of the cluster and in the suppression of cooling flows.
\end{abstract}

Keywords. Galaxies: clusters: general - cooling flows - methods: numerical

\section{Motivation and Modelling}

One of the most interesting paradigms on the physics of galaxy clusters is related to the cooling flows. It was expected that the intracluster medium (ICM) would indefinitely cool down and be pulled towards the core of the cluster by gravity. As density increases inwards cooling would be more efficient. However, cool cores are quite rare in galaxy clusters. Abell 426 (Perseus Cluster) is the brightest cluster in X-rays. At its core the giant galaxy NGC 1275 has been extensively studied in the past few years, revealing giant filamentary structures surrounding the central source (Conselice et al. 2001, Salome et al. 2006). The suppression of cooling flows and the rise of filaments are usually associated to giant bubbles of hot gas, possibly excited by the AGN (Fabian et al. 2003). However, numerical simulations have showed that the AGN's energy feedback is insufficient to reduce the cooling flow effects to the observed values (Gardini 2007), as well as inefficient in creating 


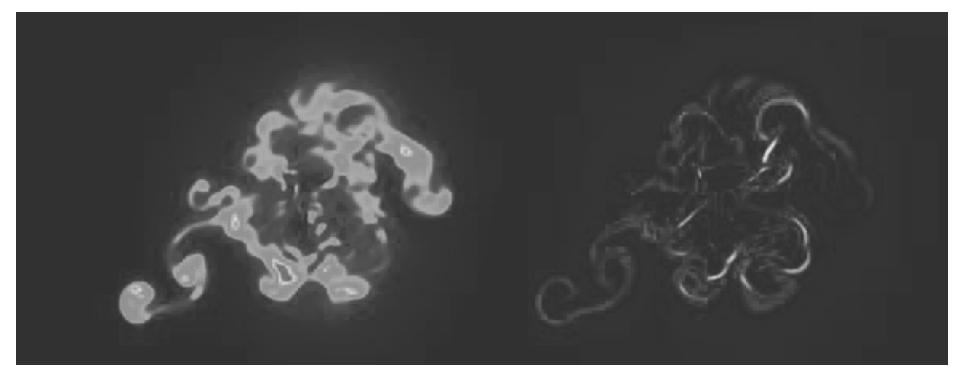

Figure 1. Density (left) and magnetic energy (right) maps for the simulated cluster core with active star formation.

isotropic distribution of filaments, as seen in NGC 1275, and additional mechanisms are needed.

In this work, we assume that $\mathrm{SNe}$ and turbulence excited by mergings may be suplementary energy feedback sources in galaxy clusters. To test this hypothesis, we perfomed a number of 2.5D magneto-hydrodynamical (MHD) simulations including turbulent motions at the central region. We implemented the problem in the well-tested GodunovMHD code studied in Falceta-Gonçalves, Kowal \& Lazarian (2008). The simulations were performed solving the set of ideal MHD equations, in conservative form, including turbulence. Gravity is implemented as a Navarro-Frenk-White potential for the distribution of dark matter. The turbulent energy was injected at a rate of $10^{56} \mathrm{ergs} / \mathrm{Myr}$, equivalent to $\sim 10^{-1} \mathrm{SN} / \mathrm{yr}$.

\section{Results and Conclusions}

Fig. 1 shows the density and magnetic energy maps. As a consequence of the $\mathrm{SNe}$ shocks, acoustic waves propagate outwards. Interactions of shocked material form filamentary structures that are confined by magnetic fields. The plasma flowing outwards also drags the magnetic energy as the loops and archs expands.

Initially, a strong cooling flow is observed in the simulation, due to the cooling and gravitaty. From the simulations, we obtained an initial infall rate of about $600 \mathrm{M}_{\odot} / \mathrm{yr}$. The effect of turbulence in the core counter balanced the cooling flow. The turbulence is responsible for both an increase in thermal and wave/kinetic pressures that prevent the cooling flow at later stages of the run. The infall ceases after $\sim 10 \mathrm{Myr}$. This effect may be responsible for the absence of cooling flows in several observed galaxy clusters.

In this work we presented preliminary results of the role of MHD turbulence in the formation of large scale structures in central galaxies of clusters. Here we assumed that SNe explosions in the starburst regions of clusters of galaxies trigger shocks that propagate outwards, dragging material and heating the medium. The numerical simulations have shown that most of the peculiar structures observed in Perseus A galaxy may be explained in this scenario. Also, the energy input of SNe occurs at a rate that is compatible with a starburst region.

\section{References}

Conselice, C. J., Gallagher, J. S., \& Wyse, R. F. G. 2001, AJ 122, 2281

Fabian, A. C., Sanders, J. S., Allen, S. W., et al. 2003, MNRAS 344, 43

Gardini, A. 2007, A\&A 464, 143

Salomé, P., Combes, F., Edge, A. C., et al. 2006, A\& A 454, 437 